\title{
Selegiline in ADHD Adults: Plasma Monoamines and Monoamine Metabolites
}

\author{
Monique Ernst, M.D., Ph.D., Laura L. Liebenauer, M.A., Daniel Tebeka, Peter H. Jons, B.A., \\ Graeme Eisenhofer, Ph.D., Dennis L. Murphy, M.D., and Alan J. Zametkin, M.D.
}

\begin{abstract}
Plasma monoamines and monoamine metabolites were assessed before and during selegiline treatment in adults with attention deficit/hyperactivity disorder (ADHD). Selegiline, at low dose, is a selective monoamine oxidase inhibitor type $B$ (MAOI-B). After 2-week placebo baseline, 36 ADHD adults were randomized to 6-week placebo or 20 $\mathrm{mg} /$ day or $60 \mathrm{mg} /$ day selegiline, followed by 2-week posttreatment placebo. Twenty-seven subjects continued into a 6-week 20-mg/day or 60-mg/day selegiline period. Behavioral variables included self-rated scores on the Conners' Abbreviated Teacher Rating Scale (ConnersATRS) and performance on a Continuous Performance Task (CPT). Plasma samples were assayed for amines
\end{abstract}

KEY WORDS: ADHD; MAOI; Plasma monoamines; Monoamine metabolites; Attention; Hyperactivity; Selegiline

The monoamine systems have been implicated in the pathophysiology of ADHD, but their relative roles are still unclear (Shenker 1992). The clinical and theoretical advantage of studying selegiline stems from its dosedependent differential effects on monoamine systems. Selegiline is a selective monoamine oxidase inhibitor type B (MAOI-B) at low doses (10-15 mg/day) and becomes a mixed MAOI-A and MAOI-B at higher doses

From the Child Psychiatry Branch (ME, LLL, DT, PHJ, AJZ), Clinical Neuroscience Branch, NINDS (GE), and Laboratory of Clinical Science, (DLM) National Institute of Mental Health, Bethesda, Maryland.

Address correspondence to: Monique Ernst, M.D., Ph.D., Brain Imaging Section, National Institute of Drug Abuse (NIDA), Intramural Research Program, P.O. Box 5180, Baltimore, MD 21224.

Received August 14, 1996; revised October 14, 1996; accepted October 17, 1996. (dopamine, norepinephrine, epinephrine), precursor (DOPA), and metabolites (HVA, DOPAC, DHPG, normetanephrine, metanephrine, 5-HIAA). Selegiline produced dose-dependent changes in monoamine metabolites and DOPA plasma levels. Dopaminergic indices were associated with ADHD symptom severity (Conners-ATRS) and noradrenergic indices with CPT performance. Serotonergic metabolism, challenged by selegiline, correlated with clinical changes. These findings support a multisystem dysfunction underlying $A D H D$ pathophysiology. [Neuropsychopharmacology 16:276-284, 1997] Published 1997 by Elsevier Science Inc.

(Heinonen et al. 1989; Knoll 1989). Because monoamine oxidase type A enzyme (MAO-A) preferentially metabolizes norepinephrine and serotonin, MAO-B phenylethylamine, and perhaps dopamine (Fuller 1968; Johnston 1968; Goridis and Neff 1971), the study of selegiline at two dose levels permits the examination of the clinical effects of changes in norepinephrine and serotonin metabolism separately from those of phenylethylamine metabolism.

The purpose of this study was twofold. The first one was to test the hypotheses of dose-related changes in plasma levels of monoamines and metabolites (see Table 1 for abbreviations). At low dose, selegiline would produce (1) increased plasma levels of DA, decreased levels of DA's deaminated metabolites (HVA and DOPAC), and decreased levels of DOPA, the latter change reflecting negative feedback following accumulation of $\mathrm{DA}_{\text {; }}(2)$ small increases in plasma levels of NE and its demethylated metabolites metanephrines (free and conjugated NMN and MN); and (3) no effect on the 
Table 1. Abbreviations of Biochemical Measures

\begin{tabular}{|c|c|c|c|}
\hline \multicolumn{2}{|c|}{ Monoamines and Metabolites } & \multicolumn{2}{|r|}{ Enzymes } \\
\hline DOPA & 3,4-Dihydroxyphenylalanine & $\mathrm{MAO}$ & Monoamine oxidase \\
\hline $\mathrm{DA}$ & Dopamine (3,4-dihydroxyphenylethylamine) & COMT & Catechol-O-methyltransferase \\
\hline NE & Norepinephrine (noradrenaline) & & \\
\hline EPI & Epinephrine (adrenaline) & & \\
\hline NMN & Normetanephrine & & \\
\hline $\mathrm{MN}$ & Metanephrine & & \\
\hline DOPAC & 3,4-Dihydroxyphenylacetic acid & & \\
\hline DHPG & 3,4-Dihydroxyphenyl (ethylene)glycol & & \\
\hline HVA & Homovanillic acid (4-hydroxy-3-methoxy-phenylacetic acid) & & \\
\hline 5-HIAA & 5-Hydroxyindoleacetic acid & & \\
\hline
\end{tabular}

serotonin metabolite 5-HIAA. At high dose selegiline would produce larger effects on DA and NE systems and decrease plasma levels of 5-HIAA.

The second goal was to explore associations between biochemical and behavioral variables that would provide testable hypotheses of the respective roles of dopaminergic, noradrenergic, and serotoninergic systems in ADHD pathophysiology.

\section{METHODS}

\section{Subjects}

Subjects were recruited by advertising in local health newsletters distributed through a national support group for parents of ADHD children. Subjects were included in the study after determination that criteria were met following a telephone interview and a 3-hour screening visit at the National Institutes of Health (NIH) Clinical Center. The rejection rate of potential subjects reached about $75 \%$. Diagnosis of ADHD required consensus of two psychiatrists board certified in general psychiatry and in child and adolescent psychiatry and well-experienced in evaluating ADHD in adults. As part of a study of the efficacy and safety of selegiline in treating ADHD in adults (Ernst et al. 1996), 36 carefully screened ADHD adults (age $37.6 \pm 7.1$ years; 24 men and 12 women) were randomized to double-blind placebo $(n=$ $11)$, daily $20 \mathrm{mg}(n=11)$ or daily $60 \mathrm{mg}(n=14)$ selegiline treatment for a 6-week period. The 6-week treatment was preceded by a 2 -week single-blind placebo baseline period and followed by a 2-week single-blind placebo posttreatment period. Twenty-seven subjects continued into an additional 6-week treatment. The subjects who had been initially randomized to placebo were subsequently randomized to either low-dose or high-dose treatment. Those initially randomized to low-dose selegiline were assigned to high-dose treatment; conversely, subjects initially randomized to lowdose were subsequently assigned to high-dose treatment. Figure 1 summarizes the distribution of subjects as a function of drug condition. The double-blind con-

\section{SAMPLE DISTRIBUTION}

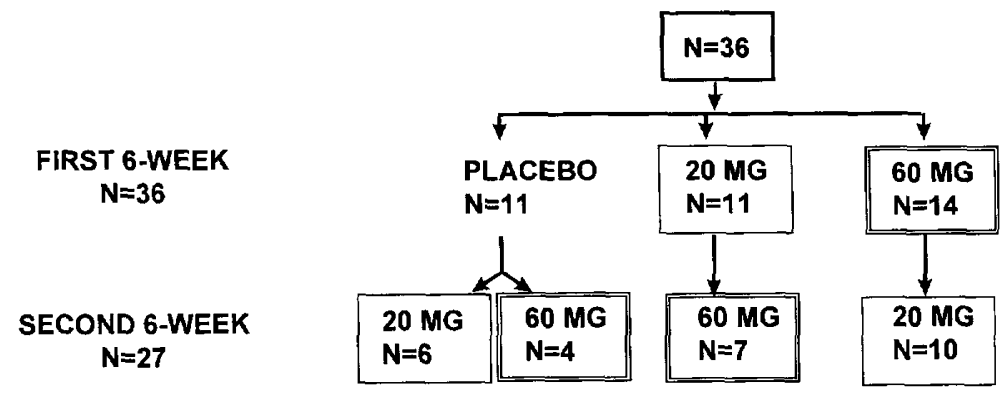

Figure 1. Distribution of patients as a function of drug condition. The first 6-week trial consisted of a three-arm doubleblind design with 11 patients randomly assigned to placebo, 11 to low-dose (20 mg), and 14 to high-dose (60 mg) selegiline. Twenty-seven of the initial 36 patients completed the crossover subsequent 6-week trial, 11 being assigned to high-dose and 16 to low-dose. A total of 27 patients completed a 6-week trial of low-dose selegiline, and a total of 25 patients completed a 6-week trial of high-dose selegiline. Of the 27 low-dose patients and 25 high-dose patients, 6 and 5, respectively, responded to initial baseline placebo and were not included in the analyses of behavioral variables. 
dition was maintained throughout the drug trial. A total of 27 subjects were randomized to low-dose selegiline treatment at any point of the trial, and 25 to high dose. HVA and 5-HIAA plasma levels were assayed in all 36 subjects, and the other biochemical measures were assayed in a subsample of 15 subjects ( 2 randomized initially to placebo and subsequently to low-dose and high-dose; 4 first randomized to low-dose and 9 to high-dose treatment).

All subjects met at least 8 of 14 ADHD DSM-III-R criteria for current and past childhood histories (American Psychiatric Association 1987) and rated above 8 on the sum of scores of the Conners' Abbreviated Teacher Rating Scale (ATRS, Conners 1969). Exclusion criteria included any other Axis I DSM-III-R psychiatric disorders assessed by the Schedule for Affective Disorders and Schizophrenia (Endicott and Spitzer 1978), major medical problems, and medical contraindications to MAOIs. Subjects were free of any medication for a minimum of 3 to 4 weeks before entry in the study. This protocol was approved by the NIMH Intramural Review Board. After complete description of the study to the subjects, written informed consent was obtained.

\section{Procedure}

Placebo and selegiline tablets were identical in appearance and were given on a twice-a-day schedule. Dosage was titrated over 10 days to the optimal dose (4 tablets in the morning and 8 tablets in the afternoon). All subjects followed the standard low-monoamine diet throughout the study (McCabe 1986). Tablets were supplied every 2 weeks, when patients came to the clinic for behavioral and blood pressure assessments. At each visit, patients brought back their medication bottles, and remaining tablets were counted to assess compliance. Behavioral measures (10-item Conners' ATRS; Conners 1969; Visual Continuous Performance Task) and blood samples were obtained in a morning clinic visit (between 7:30 A.M. and 9:30 A.M.) at the end of each of the following periods: initial 2-week placebo baseline, first 6-week treatment, and second 6-week treatment. Patients were asked not to eat or drink after midnight prior to these visits to decrease variability in plasma measures. Plasma samples were obtained after subjects had remained seated for a minimum of 10 minutes.

The Conners' ATRS (Conners 1969) was adapted to our adult sample (e.g., "disturbs other children" replaced by "disturbs others") and was self-rated by the patients. The sum of scores (range 0-30) was used for analysis. The continuous performance task (CPT) consisted of a 30-minute random presentation of degraded numbers, with an auditory distractor of a recording of numbers read to the subject through headphones. Subjects were asked to press a button each time they saw a 1 followed by a 9 . Number of correct responses within a specified latency ("hits"), number of late correct responses ("lates"), number of false responses ("incorr"), and mean reaction time to correct responses ("RT") were recorded.

Plasma was obtained from venous blood samples collected in tubes containing EDTA. Plasma samples were stored at $-80^{\circ} \mathrm{C}$ until assayed. Plasma concentration of the monoamines and their metabolites were estimated by high performance liquid chromatography (HPLC) with electrochemical detection. A pre-extraction procedure using alumina was employed for the catacholamines (Eisenhofer et al. 1986; Murphy et al. 1991), and a pre-extraction on solid-phase ion-exchange columns was used for the metanephrine and normetanephrine assays (Lenders et al. 1993).

\section{Analysis of Results}

Because there was no significant effect of order (first 6-week treatment versus second 6-week treatment) on biochemical and behavioral variables, data collected from both low-dose treatment groups and both highdose treatment groups were pooled into single combined low-dose versus high-dose samples. The baseline value used was that obtained at the end of the 2-week initial placebo baseline period in all cases.

Two-way repeated-measures analyses of variance (ANOVA) with time (baseline vs. treatment) as a withinsubjects factor and drug (low dose vs. high dose) as a between-subjects factor were used to assess the effects of selegiline on biochemical and behavioral variables.

Pearson correlation coefficients were used to assess the relationships between biochemical and behavioral variables at baseline and during selegiline treatment. Because there is no evidence that the same biochemical mechanisms mediate the placebo response and the response to active treatment, these correlations were performed on the subsample of 24 subjects who still met the criteria of entry into the study at the end of the initial 2-week placebo baseline period (sum of scores on the Conners' ATRS > 8). In this group of 24 subjects, 20 were assigned to high-dose selegiline treatment and 21 to low-dose treatment at any point of the trial. The remaining 12 subjects who improved during the first 2-week placebo were kept throughout the study for three reasons: (1) The length of the study was thought to be sufficient to allow for the placebo effect to subside and unmask the effect of the active drug; (2) we hoped to be able to characterize better the placebo response in ADHD adults; and (3) the statistical power for the analysis of biochemical measures was increased by the enlarged sample size thanks to the inclusion of placebo responders.

All statistical tests were two-tailed. Statistical significance was set at the 0.01 level to account for multiple comparisons and to reduce Type I errors. Because of the 
Table 2. Monoamines and Metabolites Plasma Levels (pmole/ml) at Baseline and after 6-Week 20-mg/day and 60-mg/day Selegiline Treatment (comparison by one-way repeated-measures ANOVA)

\begin{tabular}{|c|c|c|c|c|c|c|c|c|c|c|}
\hline \multicolumn{6}{|c|}{ High-Dose Selegiline } & \multicolumn{5}{|c|}{ Low-Dose Selegiline } \\
\hline & \multicolumn{2}{|c|}{ Baseline } & \multicolumn{2}{|c|}{ Treatment } & \multirow[b]{2}{*}{$p$} & \multicolumn{2}{|c|}{ Baseline } & \multicolumn{2}{|c|}{ Treatment } & \multirow[b]{2}{*}{$p$} \\
\hline & $n$ & Mean \pm SD & $n$ & Mean \pm SD & & $n$ & Mean \pm SD & $n$ & Mean \pm SD & \\
\hline DOPA & 14 & $1,599 \pm 622$ & 14 & $1,273 \pm 313$ & .026 & 14 & $1,671 \pm 623$ & 14 & $1,444 \pm 453$ & .057 \\
\hline $\mathrm{DA}$ & 9 & $95 \pm 91$ & 9 & $103 \pm 54$ & NS & 9 & $87 \pm 93$ & 9 & $66 \pm 47$ & NS \\
\hline DOPAC & 14 & $1,730 \pm 790$ & 14 & $701 \pm 310$ & .001 & 13 & $1,698 \pm 831$ & 13 & $996 \pm 609$ & .011 \\
\hline HVA & 23 & $31 \pm 9$ & 23 & $19 \pm 7$ & .000 & 24 & $36 \pm 17$ & 24 & $27 \pm 12$ & .013 \\
\hline $\mathrm{NE}$ & 14 & $415 \pm 153$ & 14 & $396 \pm 89$ & NS & 14 & $412 \pm 157$ & 14 & $473 \pm 264$ & NS \\
\hline DHPG & 14 & $969 \pm 150$ & 14 & $373 \pm 165$ & .000 & 14 & $981 \pm 136$ & 14 & $547 \pm 231$ & .000 \\
\hline NMN & 14 & $1,476 \pm 397$ & 14 & $5,096 \pm 2,017$ & .000 & 14 & $1,489 \pm 382$ & 14 & $3,724 \pm 1,461$ & .000 \\
\hline EPI & 11 & $77 \pm 64$ & 11 & $69 \pm 47$ & NS & 11 & $71 \pm 65$ & 11 & $59 \pm 35$ & NS \\
\hline $\mathrm{MN}$ & 14 & $817 \pm 258$ & 14 & $2,253 \pm 2,546$ & .055 & 14 & $839 \pm 247$ & 14 & $1,234 \pm 652$ & .008 \\
\hline 5-HIAA & 24 & $16 \pm 5$ & 24 & $15 \pm 3$ & .079 & 25 & $16 \pm 2$ & 25 & $16 \pm 3$ & NS \\
\hline
\end{tabular}

NS, nonsignificant, $p>.10$.

exploratory nature of the assessment of the relationships between behavioral and biochemical variables, a more stringent correction factor for multiple tests was not applied. Significances of up to $p=.10$ are presented as trend levels.

\section{RESULTS}

\section{Dose-Related Changes in Plasma Levels of Monoamines and Metabolites}

Plasma levels of monoamines and metabolites at baseline and during treatment are presented in Table 2 . The mean $( \pm \mathrm{SD}$ ) daily selegiline dose for the last week of treatment was $0.28 \pm 0.05 \mathrm{mg} / \mathrm{kg} /$ day for the low-dose and $0.79 \pm 0.13 \mathrm{mg} / \mathrm{kg} /$ day for the high-dose groups.

Selegiline, at both low and high doses, produced significant changes in plasma levels of catecholamines and their metabolites: decreased level of monoamine precursor: DOPA $(14 \%$ decrease; $\mathrm{df}=1,26 ; f=10.67 ; p=$ .003 ); decreased levels of deaminated metabolites (MAO activity) of DA and NE; DOPAC ( $46 \%$ decrease; $\mathrm{df}=$ $1,25 ; F=28.6 ; p<.0001)$, HVA $(28 \%$ decrease; $\mathrm{df}=1,45$; $F=29.43 ; p<.0001)$, DHPG ( $54 \%$ decrease; $\mathrm{df}=1,26 ; F=$ 443.1; $p<.0001)$; and increased levels of demethylated metabolites (COMT activity) of NE: NMN ( $57 \%$ increase; $\mathrm{df}=1,26 ; F=97.8 ; p<.001)$, and $\mathrm{MN}(117 \%$ increase; $\mathrm{df}=1,26 ; F=7.0 ; p<.02)$. All changes were greater in the high-dose group than in the low-dose group, and significantly so for levels of DHPG $(p=.008)$ and at a trend level for HVA $(p=.038)$.

DA, NE, EPI, and 5-HIAA were not significantly altered by selegiline treatment.

\section{Correlations between Biochemical and Behavioral Variables}

Behavioral data are reported elsewhere (Ernst et al. 1996). Baseline and means ( \pm SD) of behavioral measures for the high-dose and low-dose groups are presented in Table 3. Briefly, significant reductions in severity of symptoms from baseline to end of treatment

Table 3. Behavioral Measures at Baseline and after 6-Week 20-mg/day and 60-mg/day Selegiline Treatment

\begin{tabular}{|c|c|c|c|c|c|c|c|c|c|c|}
\hline \multicolumn{6}{|c|}{ High-Dose Selegiline } & \multicolumn{5}{|c|}{ Low-Dose Selegiline } \\
\hline & \multicolumn{2}{|c|}{ Baseline } & \multicolumn{2}{|c|}{ Treatment } & \multirow[b]{2}{*}{$p$} & \multicolumn{2}{|c|}{ Baseline } & \multicolumn{2}{|c|}{ Treatment } & \multirow[b]{2}{*}{$p$} \\
\hline & $n$ & Mean $\pm S D$ & $n$ & Mean $\pm S D$ & & $n$ & Mean \pm SD & $n$ & Mean $\pm S D$ & \\
\hline Conners & 20 & $13.0 \pm 3.1$ & 20 & $10.0 \pm 5.0$ & .008 & 21 & $12.5 \pm 3.2$ & 19 & $10.4 \pm 3.9$ & .007 \\
\hline CPT-hits & 20 & $84.3 \pm 20.4$ & 17 & $89.6 \pm 7.1$ & NS & 18 & $85.2 \pm 20.2$ & 17 & $91.4 \pm 6.5$ & NS \\
\hline CPT-lates & 20 & $8.3 \pm 17.5$ & 17 & $2.8 \pm 3.0$ & NS & 21 & $7.7 \pm 17.2$ & 17 & $2.2 \pm 2.3$ & 0.90 \\
\hline CPT-incorr & 20 & $7.0 \pm 18.5$ & 19 & $1.8 \pm 2.9$ & NS & 21 & $6.6 \pm 20.6$ & 19 & $1.8 \pm 2.9$ & NS \\
\hline CPT-RT & 20 & $50.3 \pm 11.1$ & 17 & $44.8 \pm 6.4$ & NS & 21 & $48.6 \pm 9.2$ & 17 & $44.5 \pm 7.1$ & NS \\
\hline
\end{tabular}

NS, nonsignificant, $p>.10$. 


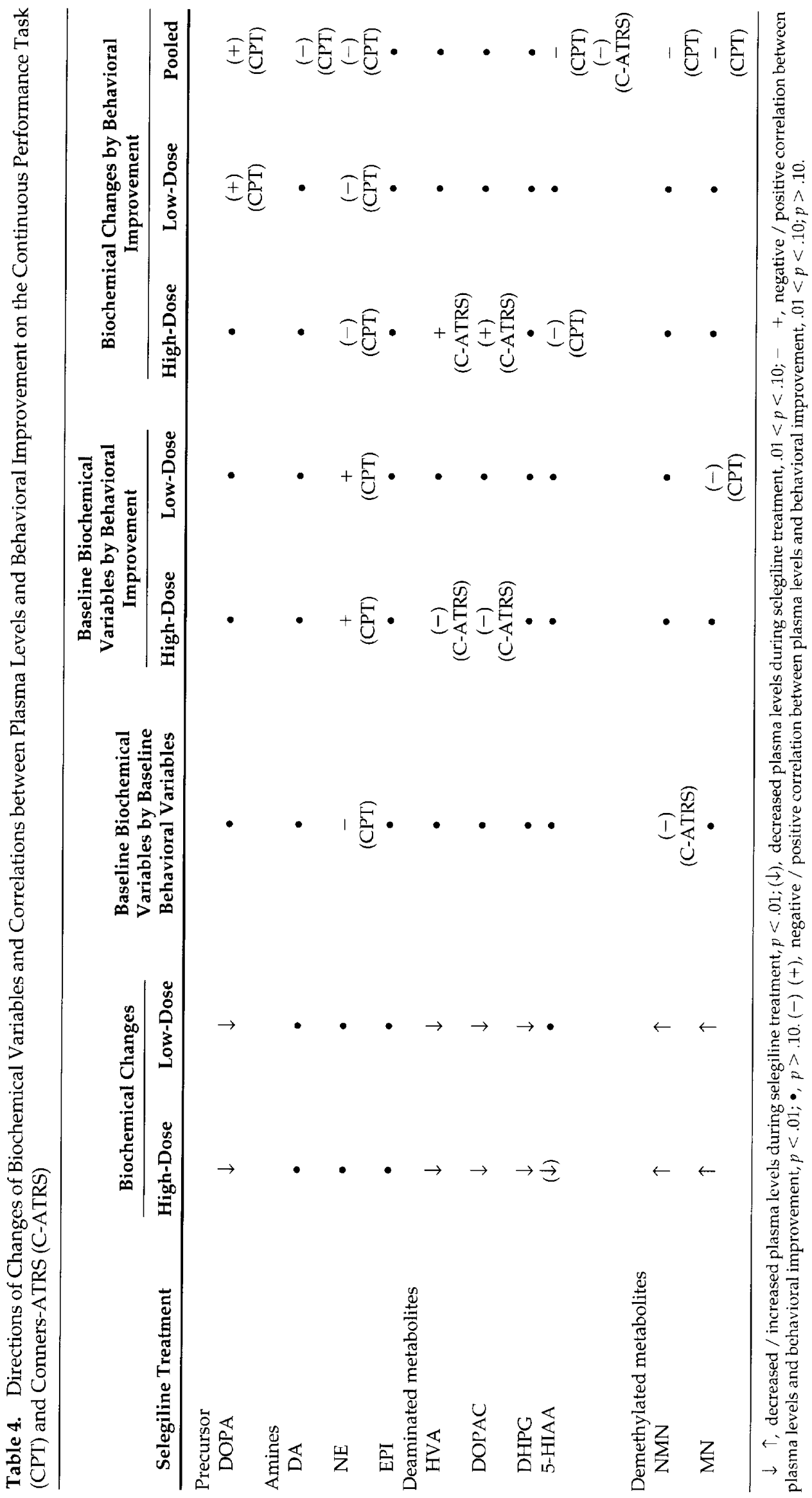


were shown in the low-dose and high-dose selegiline groups. However, these changes were not significantly different from the nonsignificant decreases occurring during placebo condition. Analyses of the behavioral data in relation to biochemical variables were conducted in the subsample of 24 subjects who continued to meet the study entry criteria at the end of the initial 2-week placebo baseline.

Baseline Biochemical and Baseline Behavioral Measures (Table 4). Only the noradrenergic indices and none of the dopaminergic ones were associated with behavioral variables. Lower baseline NE levels correlated with better CPT performance at baseline on CPT-hits $(n=10 ; r=$ $-0.78, p=.006)$, CPT-lates $(n=10 ; r=0.77, p=.006)$, and at a trend level on CPT-RT $(n=10 ; r=0.70, p=.021)$. In addition, there was a trend for lower baseline NMN levels that can be associated with lower symptom severity at baseline on Conners'-ATRS ( $n=10, r=0.55, p=.10)$.

Baseline Biochemical Measures and Changes in Behavioral Variables (Table 4). In the high-dose group, for noradrenergic measures, higher NE levels at baseline correlated with CPT performance improvement after selegiline treatment on all CPT variables (CPT-hits $n=$ 9, $r=0.83, p=.004$; CPT-lates: $n=9, r=-0.79, p=$ .008; CPT-RT: $n=9, r=-0.79, p=.009$ ), except for CPT-incorr $(n=9, r=-0.41, p=.30)$. For dopaminergic measures, lower DOPAC and HVA baseline levels tended to correlate with improvement on the Conners' ATRS (DOPAC: $\mathrm{n}=10, r=0.60, p=.067$, and HVA: $\mathrm{n}=$ $19, r=0.44, p=.061)$. For serotoninergic measure, baseline 5-HIAA was not associated with behavioral changes during high-dose treatment.

In the low-dose group, for noradrenergic measures, as with the high-dose group, higher NE levels at baseline predicted CPT performance improvement after selegiline treatment on all CPT variables (CPT-hits: $n=8$, $r=0.77, p=.02$; CPT-lates: $n=8, r=-0.82, p=.01$; CPT-RT: $n=8, r=-0.79, p=.017$ ) except CPT-incorr $(n=8, r=-0.15, p=.73)$. Lower MN baseline levels tended to predict improvement on CPT performance (CPT-RT: $n=8, r=0.73, p=.030$ ). For dopaminergic measures, none of the baseline measures were associated with behavioral changes at low-base selegiline. For serotoninergic measure, baseline 5-HIAA was not associated with behavioral changes during low-dose treatment.

Biochemical Changes and Behavioral Changes during Selegiline Treatment (Table 4). In the high-dose group, changes in noradrenergic measures were defined by the magnitude of decreases in NE level, which tended to be associated with improvement on CPT performance (CPT-hits: $\mathrm{n}=9, r=0.70, p=.03$; CPT-RT: $\mathrm{n}=9$, $r=0.67, p=.049$; CPT-lates: $\mathrm{n}=9, r=0.70, p=.034$ ). For changes in dopaminergic measures, the degree of increases in HVA level was associated with improvement on Conners' ATRS $(n=16, r=-0.73, p=.0008)$. There was a similar trend for DOPAC levels $(n=10, r=$ $-0.54, p=.10)$. As far as changes in serotoninergic measures were concerned, levels of 5-HIAA tended to decrease with reduction in CPT-RT $(n=16, r=0.45, p=.084)$.

For the low dose group, changes in noradrenergic measures were characterized by a trend for NE levels to decrease with CPT improvement (NE * CPT-incorr: $n=$ $8, r=0.64, p=.093$ ). Changes in dopaminergic measures were characterized by the magnitude of increases in DOPA levels, which tended to correlate with improvement on CPT-RT performance $(n=8, r=0.79, p=$ .018). Changes in 5-HIAA did not correlate with behavioral variables.

For the combined low-dose and high-dose groups (the combined group was used for this analysis as both biochemical and clinical variables are affected by drug dosage, and the pooled sample provided greater statistical power) changes in noradrenergic measures revealed that the magnitude of reductions in $\mathrm{NE}, \mathrm{NMN}$, and MN levels tended to be associated with CPT improvement (ranges: $n=14-17 ; r=0.55-0.45 ; p=.02-.07$ ). Changes in dopaminergic measures were characterized by the magnitude of reductions in DA levels, which tended to be associated with CPT improvement (CPT$\mathrm{RT}: n=12 ; r=0.58 ; p=.046$ ). As far as serotoninergic changes were concerned, the extent of reductions in 5-HIAA plasma levels was associated with CPT improvement (CPT-RT: $n=30 ; r=0.50 ; p=.007$ ) and also tended to be associated with reduction of symptom severity on the Conners'-ATRS ( $n=33 ; r=0.32 ; p=.071$ ).

\section{DISCUSSION}

Plasma monoaminergic measures to assess cerebral neuronal activity and to understand the mechanism of action of psychotropic drugs should be used with caution because they reflect both peripheral and central metabolism. However, considerable data from studies in animals and humans indicate that MAOIs in general and selegiline specifically alter many biochemical measures in a similar way centrally and peripherally (Murphy et al. 1981; Sandler 1982; Kopin 1985; Celada et al. 1990).

Selegiline produced the expected changes in monoamine metabolites, that is, it decreased deaminated metabolites (DOPAC, HVA, DHPG) through direct inhibition of MAO enzymes and increased demethylated metabolites (NMN and MN) as the alternate COMT pathway of monoamines metabolism. At high dose of selegiline treatment, the order of magnitude of DHPG and HVA reductions (62\% and $39 \%$, respectively) was consistent with that reported in the cerebrospinal fluid (CSF) following $60 \mathrm{mg} /$ day treatment with selegiline in de- 
pressed elderly patients ( $51 \%$ and $21 \%$, respectively) (Sunderland et al. 1994). Of interest is the significantly stronger influence of high-dose treatment on HVA and DHPG plasma levels compared to low-dose treatment. In fact, all other biochemical measures showed larger changes with high-dose treatment, but not to a statistically significant degree. The increased NMN and MN mirrored the changes of DHPG and reflected the compensatory shift to the COMT pathway of NE and EPI metabolism. In fact, COMT reserve activity offset the loss of the deamination process, as evidenced by the absence of significant increase of DA, NE, and EPI.

\section{Dopaminergic Activity and ADHD Behavior}

Baseline ADHD severity and CPT performance were independent of baseline dopaminergic measures. This finding is consistent with the absence of differences of HVA CSF levels between ADHD and normal controls in adults (Reimherr et al. 1984) and in children (Shetty and Chase 1976). However, reports in the literature are discordant. Lower CSF levels of HVA were reported in children with ADHD than in controls (Shaywitz et al. 1977), whereas higher CSF levels of HVA were found to be associated with worse hyperactivity in children with ADHD (Castellanos et al. 1994). These discrepancies may reflect differences in sample characteristics and methodologies.

With regard to the relationship of baseline dopaminergic function and changes in behavior produced by MAOI, lower levels of the deaminated DA metabolites (HVA and DOPAC) tended to predict improvement in behavior on Conners'-ATRS during high-dose selegiline treatment. This finding is consistent with the report by Reimherr et al. (1984) of a trend for an association between high HVA CSF levels and lack of response to methylphenidate treatment in adults with ADHD. However, opposite results also have been found in ADHD children where higher baseline CSF HVA predicted improvement in hyperactive behavior during stimulant treatment (Castellanos et al. 1996). Comparisons of findings of biochemical and behavioral associations during studies of stimulants and studies of MAOIs call for caution because of the differences in the pharmacological mechanisms of action of these agents and in the types of biological (CSF vs. plasma) and clinical samples (age, comorbidity). Low baseline levels of HVA may reflect either less availability of intracellular DA to be degraded by MAOs or reduced MAO activity. Our finding would suggest that ADHD individuals with low DA tone at baseline benefit more substantially from treatments that increase DA activity. The absence of correlation between HVA and behavior at baseline may indicate the heterogeneity of the pathophysiology of ADHD, or various individual sensitivity to or compensatory mechanisms for low DA activity.
Finally, the smaller the HVA reductions following high-dose selegiline treatment, the more improved ADHD symptoms on the Conners'-ATRS. This finding actually reflected larger inhibition of HVA synthesis in subjects with higher HVA baseline levels [HVA (baseline) * HVA (changes): $r=-0.80, p<.0001]$. Again, subjects with high HVA baseline levels (and large selegiline-related HVA reductions) did not improve as much as those with low HVA baseline levels (and small selegiline-related HVA reductions). Shetty and Chase (1976) reported the opposite in children treated with dextroamphetamine (i.e., larger reductions in HVA CSF levels predicted greater improvement during stimulant treatment). However, the authors did not provide measures of association between baseline and treatmentrelated changes of HVA CSF levels. Comparison of findings between CSF and plasma studies should be made with caution.

Associations between behavioral changes and dopaminergic activity emerged during selegiline treatment only at high dose and not at low dose. This fact suggests that the powerful inhibition of DA deamination and the resulting enhanced dopaminergic activity are necessary to affect behavior or that the interaction of the inhibition of both MAO-B and MAO-A is more effective on behavior.

Of interest, the association of dopaminergic indices were with overall ADHD symptom severity (Conners'ATRS), and not with performance on the attention task. This finding differs from the association of noradrenergic activity with CPT performance and not with overall ADHD severity.

\section{Noradrenergic Activity and Attention}

In contrast to findings from previous reports that used monoaminergic concentration levels in the CSF (Shaywitz et al. 1977; Castellanos et al. 1996) and in 24-hour urine (Zametkin et al. 1984) of children with ADHD, noradrenergic indices were significantly associated with measures of attention in the present study (i.e., higher baseline plasma NE predicted worse performance on CPT at baseline and better improvement during treatment). However, our result is consistent with reports of significant correlations between urinary and plasma MHPG levels with behavioral improvement during desipramine treatment of ADHD children (Donnelly et al. 1989). ADHD children also have been shown to have lower urinary MHPG than controls (Shekim et al. 1979; Yu-Cun and Yu-Feng 1984). This discrepancy can be explained by the model discussed by Dienstbier (1989) indicating that good performance on cognitive tasks depended on both low basal levels of catecholamines (consistent with our findings) and high acute releases of catecholamine during the tasks (not tested herein). The contribution of NE plasma concentration to behavioral 
measures seemed to be independent of dopaminergic activity, as baseline NE did not correlate with any of the dopaminergic indices. This finding suggests a noradrenergic role in ADHD pathophysiology already proposed in earlier reports (Zametkin and Rapoport 1987; Mefford and Potter 1989; Shenker 1992). In fact, converging evidence from research with animals (Segal and Bloom 1976; Aston-Jones et al. 1984; Pineda et al. 1989) has indicated that the locus coeruleus plays a central role in attention and contributes to the brain's signal-tonoise ratio in the processing of afferent signals.

\section{Serotoninergic Activity and ADHD}

The decreased plasma levels of the serotonergic deaminated metabolite 5-HIAA during selegiline treatment correlated with selegiline-related improvement of attention on CPT in the pooled low-dose and high-dose groups and tended to correlate with a reduction of symptom severity on the Conners'-ATRS during highdose selegiline treatment. This association was independent of dopaminergic and noradrenergic activities as baseline 5-HIAA did not correlate with any of the dopaminergic or noradrenergic indices (not reported in the results). This finding suggests a role of the serotoninergic system in ADHD symptomatology, despite the paucity of such evidence in the literature (Reimherr et al. 1984; Shetty and Chase 1976; Irwin et al. 1981; Rapoport et al. 1974; Weizman et al. 1988). The association of changes in ADHD behavior with plasma levels of 5-HIAA may support the role of serotoninergic activity reported in impulsivity (Soubrie 1986).

\section{CONCLUSION}

In summary, selegiline treatment produced dose-dependent changes of monoaminergic plasma levels. Associations of behavioral variables were found with dopaminergic, noradrenergic, and serotoninergic biochemical measures. These findings support the model of a multisystem dysfunction underlying ADHD pathophysiology (Mefford and Potter 1989; McCracken 1990; Pliszka et al. 1996). Furthermore, some degree of specialization in the role of the various neurotransmitter systems in behavior also is suggested by this study: low dopaminergic tone could mediate overall symptom severity, abnormal noradrenergic tone impairment in attention, and serotoninergic activity impulsivity.

\section{ACKNOWLEDGMENTS}

We thank Kristina Hardy, Akia Talbot, and Teresa Talliner for their help in this project. This study was supported in part by a grant from Somerset Pharmaceuticals, Inc.

\section{REFERENCES}

American Psychiatric Association (1987): Diagnostic and Statistical Manual of Mental Disorders, ed 3, rev. Washington, DC: American Psychiatric Press

Aston-Jones G, Foote SJ, Bloom FE (1984): Anatomy and physiology of locus coeruleus neurons: Functional implications. In Zeigler MG, Lake CR (eds), Norepinephrine: Clinical aspects. Baltimore, Williams \& Wilkins, pp 113125

Castellanos FX, Elia J, Kruesi MJP, Gulotta CS, Mefford IN, Potter WZ, Ritchie GF, Rapoport JL (1994): Cerebrospinal fluid monoamine metabolites in ADHD boys. Psychiatr Res 52:205-316

Castellanos FX, Elia J, Kruesi MJP, Marsh WL, Gulotta CS, Potter WZ, Ritchie GF, Hamburger SD, Rapoport JL (1996): Cerebrospinal fluid homovanillic acid predicts behavioral response to stimulants in 45 boys with Attention-Deficit/Hyperactivity Disorder. Neuropsychopharmacology 14:125-137

Celada P, Sarriias MJ, Artigas F (1990): Serotonin and 5-hydroxyindoleacetic acid in plasma: Potential use as peripheral measures of MAO-A activity. J Neural Transm 32:149154

Conners CK (1969): A teacher rating scale for use in drug studies with children. Am J Psychiatr 126:152-156

Dienstbier RA (1989): Arousal and physiological toughness: Implications for mental and physical health. Psychol Rev 96:4-100

Donnelly M, Rapoport JL, Potter WZ, Oliver J, Keysor CS, Murphy DL (1989): Fenfluramine and dextroamphetamine treatment of childhood hyperactivity: Clinical and biochemical findings. Arch Gen Psychiatr 46:205212

Eisenhofer G, Goldstein DS, Stull R, Keiser HR, Sunderland T, Murphy DL, Kopin IJ (1986): Simultaneous liquidchromatographic determination of 3,4-dihydroxyphenylglycol, catecholamines, and 3,4-dihydroxyphenylalanine in plasma, and their responses to inhibition of monoamine oxidase. Clin Chem 32:2030-2033

Endicott J, Spitzer RL (1978): A diagnostic interview: The schedule for affective disorders and schizophrenia. Arch Gen Psychiatr 35:837-844

Ernst M, Liebenauer LL, Jons PH, Tebeka D, Cohen RM, Zametkin AJ (1996): Selegiline in adults with Attention Deficit/Hyperactivity Disorder: Clinical efficacy and safety. Psychopharm Bull 32:327-334

Fuller RW (1968): Influence of substrate in the inhibition of rat liver and brain monoamine oxidase. Arch Intern Pharmacodyn Ther 174:32-37

Goridis C, Neff NH (1971): Evidence for a specific monoamine oxidase B associated with sympathetic nerves. Neuropharmacology 10:557-564

Heinonen EH, Myllyla V, Sotaniemi K, Lammintausta R, Salonen JS, Anttila M, Savijarvi M, Kotila M, Rinne UK (1989): Pharmacokinetics and metabolism of selegiline. Acta Neurol Scand 126:93-99

Irwin M, Elendick K, McGloskay K, Freedman DX (1981): Tryptophan metabolism in children with attention deficit disorder. Am J Psychiatr 138:1082-1085 
Johnston JP (1968): Some observations upon a new inhibitor of monoamine oxidase in brain tissue. Biochem Pharmacol 17:1285-1297

Knoll J (1989): The pharmacology of selegiline $((-)$ deprenyl). New aspects. Acta Neurol Scand 126:83-91

Kopin IJ (1985): Catecholamine metabolism: Basic aspects and clinical significance. Pharm Rev 37:334-364

Lenders JWM, Eisenhofer G, Armando I, Keiser HR, Goldstein DS, Kopin LJ (1993): Determination of metanephrines in plasma by liquid chromatography with electrochemical detection. Clin Chem 39:97-103

McCabe BJ (1986): Dietary tyramine and other pressor amines in MAOI regimens: A review. J Am Diet Assoc 86:1059-1064

McCracken JT (1990): A two-part model of stimulant action on attention-deficit hyperactivity disorder in children. J Neuropsychiatr Clin Neurosci 3:201-209

Mefford IN, Potter WZ (1989): A neuroanatomical and biochemical basis for attention deficit disorder with hyperactivity in children: A defect in tonic adrenaline mediated inhibition of locus coeruleus stimulation. Med Hypotheses 29:33-42

Murphy DL, Pickar D, Jimerson D, Cohen NA, Garrick N, Karoum F, Wyatt RJ (1981): Biochemical indices of the effects of the selective MAO inhibitors clorgyline, pargyline and deprenyl in man. In Usdin E, Dahl SG, Gram LF, Ligjaerde O (eds), Clinical Pharmacology in Psychiatry Neuroleptic and Antidepressants Research. New York: Macmillan, pp 301-316

Murphy DL, Sims KB, Karoum F, Garrick NA, De La Chapelle A, Sankila EM, Norio R, Breakefield XO (1991): Plasma amine oxidase activities in Norrie disease patients with an X-Chromosomal deletion affecting monoamine oxidase. J Neural Transm Gen Sect 83:1-12

Pineda JA, Foote SL, Neville HJ (1989): Effects of locus coeruleus lesions on auditory, long-latency, event related potentials in monkeys. J Neurosci 9:429-432

Pliszka SR, McCracken JT, Maas JW (1996): Catecholamines in attention-deficit hyperactivity disorder: Current perspectives. J Am Acad Child Adol Psychiatr 35:264-272

Rapoport JL, Lott I, Quinn PO, Scribanic N, Murphy DL (1974): Platelet serotonin of hyperactive school age boys. Br J Psychiatr 125:138-140

Reimherr FW, Wender PH, Ebert MH, Wood DR (1984): Cerebrospinal fluid homovanillic acid and 5-hydroxyindole acetic acid in adults with attention deficit hyperactivity disorder, residual type. Psychiatr Res 11:71-78
Sandler M (1982): Catecholamine synthesis and metabolism in man: Clinical implications (with special reference to parkinsonism). Catecholamines. In Blashko $\mathrm{H}$, Muscholl E (eds), Handbook of Experimental Pharmacology, vol 33. Berlin, Springer-Verlag, pp 845-899

Segal M, Bloom FE (1976): The action of norepinephrine in the rat hippocampus: IV. The effects of locus coeruleus stimulation on evoked hippocampal unit activity. Brain Res 107:513-525

Shaywitz BA, Cohen DJ, Bowers MB (1977): CSF amine metabolites in children with minimal brain dysfunction. J Pediatr 90:67-71

Shekim WO, Dekirmenjian H, Chapel JL (1979): Urinary catecholamine MHPG excretion in minimal brain dysfunction and its modification by $d$-amphetamine. Am J Psychiatr 136:667-671

Shenker A (1992): The mechanism of drugs used to treat Attention-Deficit Hyperactivity Disorder: Focus on catecholamine receptor pharmacology. Adv Pediatr 39:337382

Shetty T, Chase TN (1976): Central monoamines and hyperkinesis of childhood. Neurology 26:1000-1006

Soubrie P (1986): Reconciling the role of central serotonin neurons in human and animal behavior. Behav Brain Sci 9:319-364

Sunderland T, Cohen RM, Molchan S, Lawlor BA, Mellow AM, Newhouse PA, Tariot PN, Mueller EA, Murphy DL (1994): High-dose selegiline in treatment-resistant older depressive patients. Arch Gen Psychiatr 51:607-612

Weizman A, Bernhout E, Weitz R, Tyano S, Rehavi M (1988): Imipramine binding of platelets of children with attention deficit hyperactivity disorder. Biol Psychiatr 23:491496

Yu-Cun A, Yu-Feng W (1984): Urinary 3-methoxy-4-hydroxyphenylglycol sulfate excretion in seventy-three school children with minimal brain dysfunction syndrome. Biol Psychiatr 19:861-870

Zametkin AJ, Rapoport JL (1987): Neurobiology of attention deficit disorder with hyperactivity: Where have we come in 50 years? J Am Acad Child Adol Psychiatr $5: 676-686$

Zametkin AJ, Brown GL, Karoum F, Rapoport JL, Langer DH, Chuang LW, Wyatt RJ (1984): Urinary Phenethylamine Response to d-Amphetamine in 12 Boys with Attention Deficit Disorder. Am J Psychiatr 141:9:10551058 